\title{
Least-squares migration for improved QI analysis, a case study from Buzios field
}

Yermek Balabekov (PGS), Sriram Arasanipalai (PGS), Nizar Chemingui (PGS)

Copyright 2017, SBGf - Sociedade Brasileira de Geofísica

This paper was prepared for presentation during the $15^{\text {th }}$ International Congress of the Brazilian Geophysical Society held in Rio de Janeiro, Brazil, 31 July to 3 August 2017.

Contents of this paper were reviewed by the Technical Committee of the $15^{\text {th }}$ International Congress of the Brazilian Geophysical Society and do not necessarily represent any position of the SBGf, its officers or members. Electronic reproduction or represent any position of the SBG, its officers of the Brazilian Geophysical Society is prohibited.

\section{Abstract}

Image quality and amplitude reliability below salt structures are extremely important for successful exploration and production. New methodologies improve the seismic data, including least-squares migration, which has emerged as a new standard in high-end imaging. It provides the level of resolution and amplitude fidelity necessary for prospect risk mitigation, reservoir characterization and well planning. This work demonstrates a well-based validation test of conventional and least-squares migrations.

\section{Introduction}

Modern seismic acquisition enables more efficient presalt exploration. Techniques are constantly being developed to improve image quality and amplitude fidelity below salt structures. It is difficult to overcome sub-salt illumination problems arising from complex salt structures. In this paper outcomes of two imaging techniques- conventional and least-squares, are compared, including the seismic amplitude response, and primary and shear velocity ratios.

\section{Area of the study}

The study area covers the Buzios field, which is the second largest presalt hydrocarbon reservoir in the Santos Basin, offshore Brazil. The field is located 230 $\mathrm{km}$ away from the coast of Rio de Janeiro, $5 \mathrm{~km}$ below the $2 \mathrm{~km}$ deep water bottom. It is estimated to contain 3058 million barrels of oil.

A large-scale evolving rejuvenation program called Santos Vision undertaken by PGS, has been ongoing since 2016. The reprocessed seismic data covers a contiguous area of approximately 50000 square kilometers, larger than the entire state of Rio de Janeiro or the country of Denmark. Underlying narrow azimuth legacy survey called BS-500 acquired over the Buzios field has been reprocessed as part of the abovementioned rejuvenation effort. Figure 1 shows a location map of the Buzios field - green outline, partially included in the Santos Vision rejuvenation project (left of the pink border). Sections A-A' and B-B" are the QC lines. W2' is

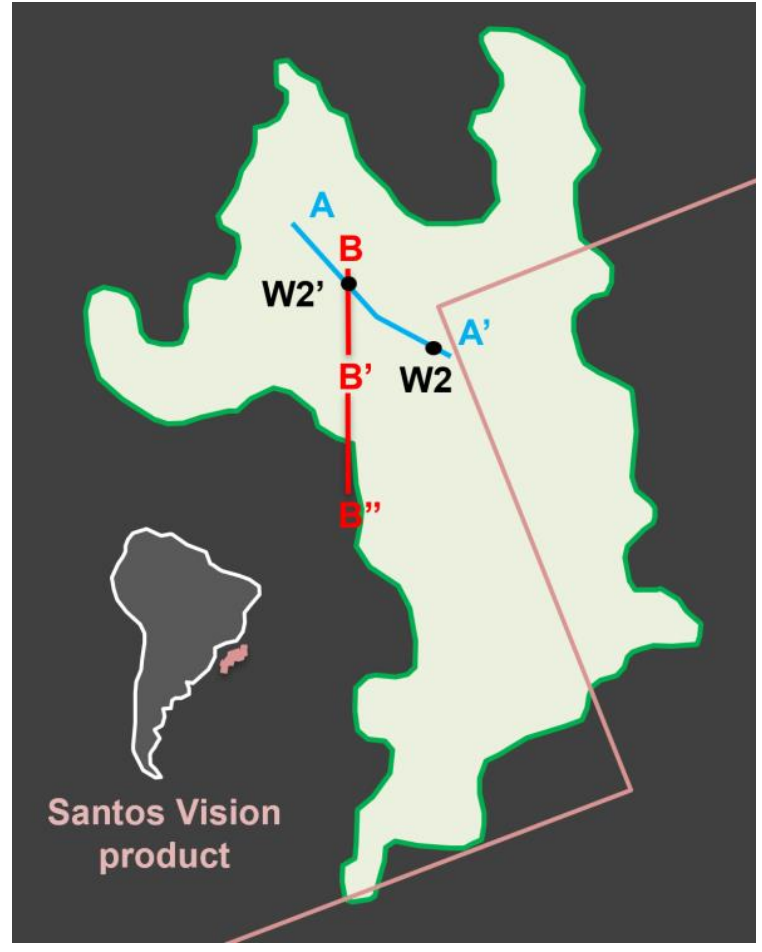

Figure 1 Schematic display of the study area

the projected calibration well, with its original position indicated as W2.

\section{The challenge}

It is common to avoid using first few degrees in the near angle range as well as far and ultra-far angles for seismic reservoir characterization in the presalt section. While they are valuable records in the postsalt section, seismic is affected most by the complex overburden and salt geometries at deeper intervals. Unfortunately, this means a sub-optimal number of angle stacks/offsets are used to describe qualitative and quantitative properties of the reservoir rocks. Presalt carbonates as a rock are known to be rigid and have a porosity much lower than the one of a conventional sandstone reservoir (cavities / karstification and extreme fracturing are not considered in this context). To minimize uncertainty in the extracted elastic-reservoir properties, it is highly desirable to broaden angle ranges that can be used for analysis, especially far angles as they contain information about pore space and fluids. In this paper we demonstrate that it is possible to stabilize and improve prestack seismic amplitudes in the presalt section. 
Complex overburden such as rugose heterogenous salt bodies and intervals with high velocity contrasts (e.g. fast carbonates or volcanic sills) distort the wavefield and affect imaging. Illumination defines the quality and completeness of the wavefield. Figure 2 shows an estimated presalt illumination metric for slices and sections corresponding to different partial stacks (Near Mid and Far angle stacks respectively 10, 20 and 30 degree mean angles). While the illumination at 10 and 20 degrees has acceptable ranges (warm colors), the 30 degree angle stack shows poor illumination in presalt section. Conventional depth migrations produce an approximation of the earth's reflectivity even with an accurate velocity model. The heterogeneity of the overburden and/or limitations of some acquisition geometries can affect the illumination and wavenumber content of the image from a conventional migration, especially in the far angles. An improved approximation of the earth's reflectivity can be obtained using a Leastsquares migration (LSM) by posing the seismic imaging problem as an inversion process (Nemeth et al., 1999).

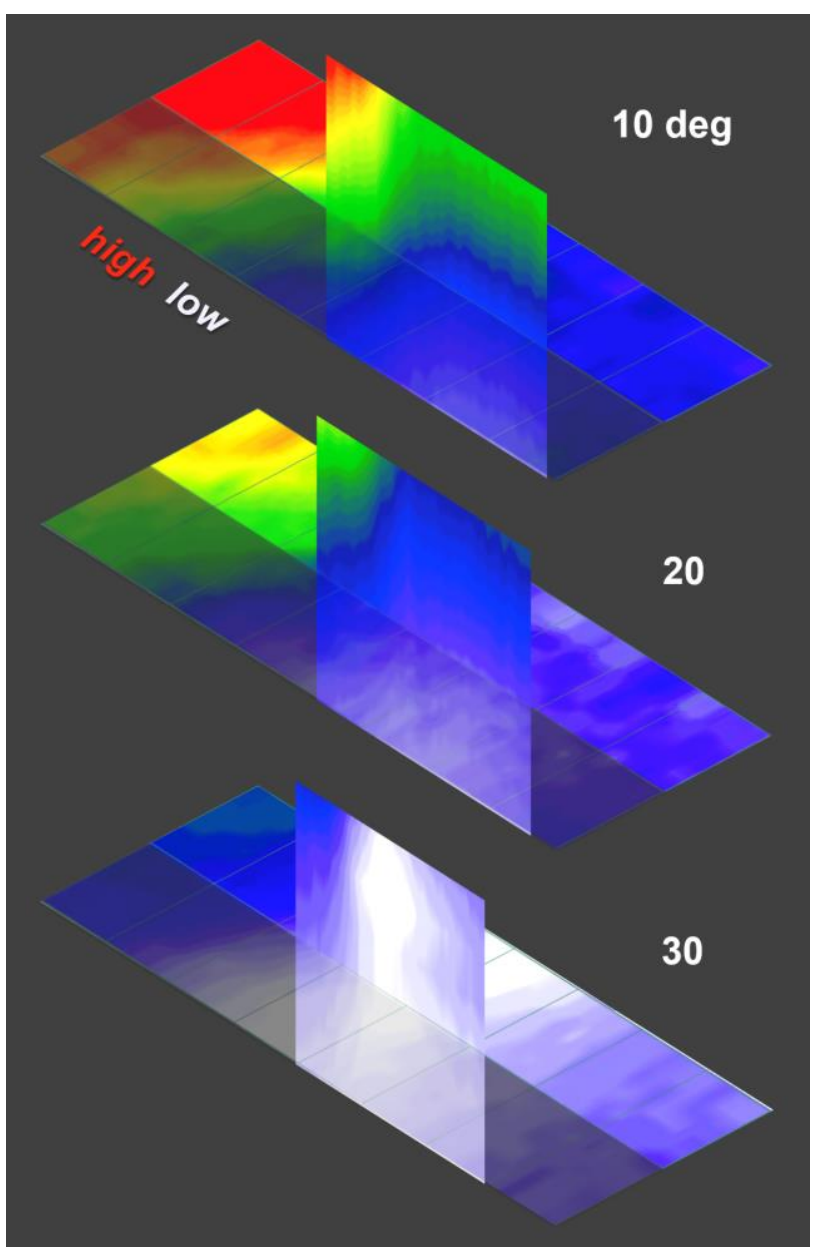

Figure 2 Estimated illumination for 10, 20 and 30 degree angle stacks. Warm colors represent good illumination and cold colors represent poor illumination. Depth slices are taken from presalt interval and sections correspond to the line shown on Figure 6 (prior to LSM).

\section{Least-squares migration}

The data-domain LSM algorithm (Lu et al., 2017) solves for the earth reflectivity by means of an iterative datafitting workflow (Figure 3) like Full Waveform Inversion $(\mathrm{FWI})$. More than one iteration of data modeling and migration are often necessary to obtain the optimal result. The engine of LSM efficiently propagates high-frequency seismic data using the detailed earth models derived from FWI. The results are high-resolution sections

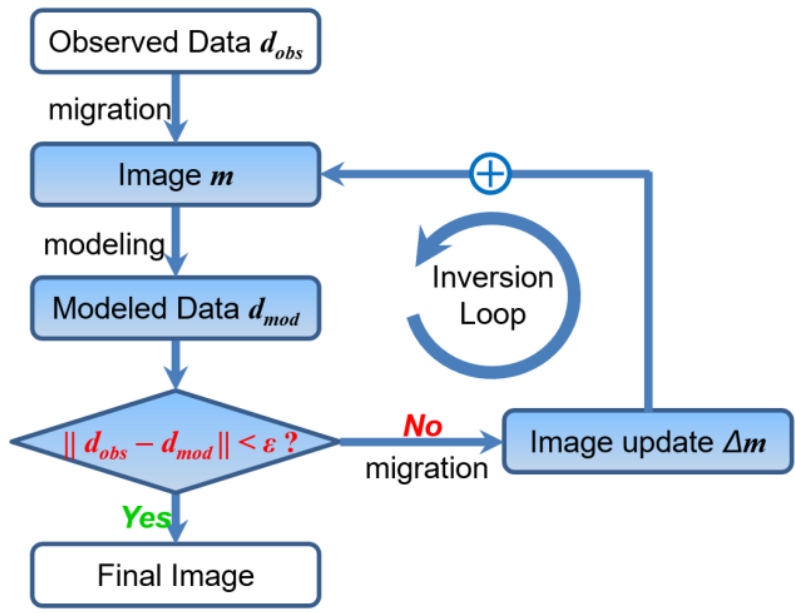

Figure 3 An illustration of the iterative, data-domain Least-squares migration workflow.

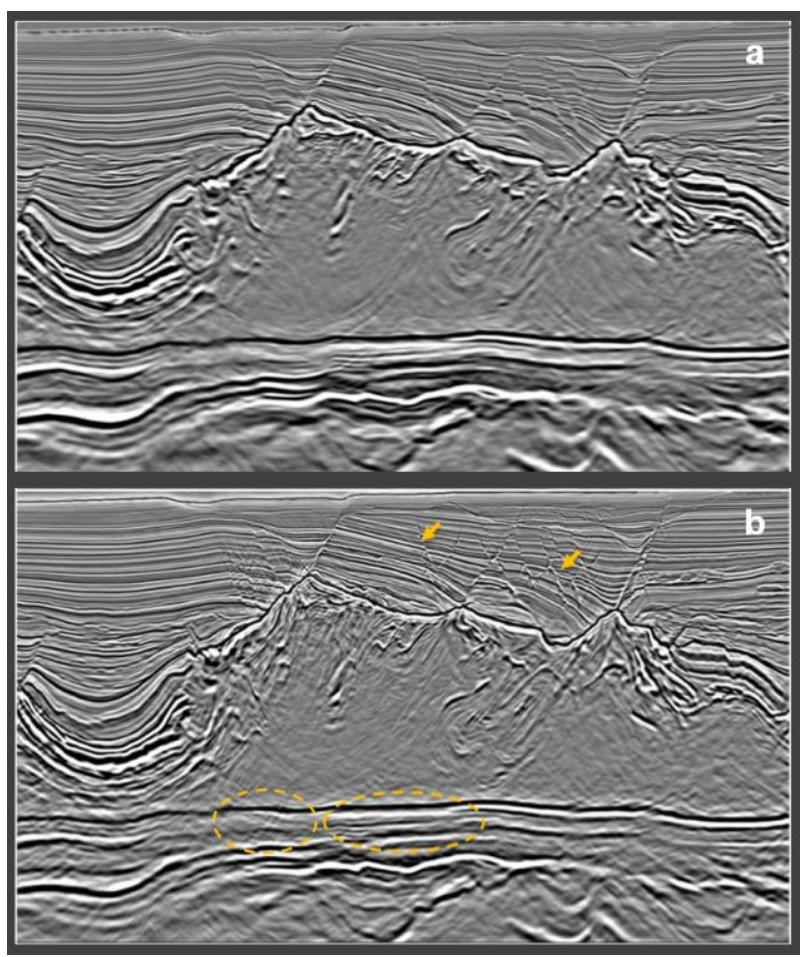

Figure 4 Conventional depth migration (a) and datadomain LSM (b) full stack. 
with balanced amplitudes, wavenumbers and reduced illumination variations. The reflectivity models have amplitudes that are more reliable and more suitable for quantitative reservoir characterization. A recent application of the data-domain LSM in the study area (Arasanipalai et al., 2019) demonstrates the benefit of the technology at all stratigraphic levels (Figure 4), but most importantly in the presalt section. Note, the improved definition of fault planes shown with arrows, intra-salt details and a better imaged presalt carbonate wedge (orange circles).

\section{Seismic resolution and AVA response}

Another example of a full stack comparison between LSM and conventional migration is shown in Figure 5. The seismic reflectors have become more continuous and more distinct. Lateral seismic character variations as well as local faulting details are now more prominent. The well W2' is a projection of the well W2 along the A-A' section. Well W2 had to be project to the nearby location because image-domain LSM was only available at that part of the volume. Both wells are positioned in similar parts of the presalt section not too distant from each other. The wellto-seismic tie analysis also proved a good correlation. To address the limitations of a conventional migration in prestack domain, an image-domain LSM using point spread functions Valenciano (2008) was used to create angle gathers for a reliable AVA response. The left picture in Figure 6 is the far stack (30 deg.) from conventional migration and the right section is a product of an imagedomain LSM. The result shows that the LSM improves resolution of the image and compensates for varying illumination with angle, providing AVA fidelity in the target section.

A set of gathers around the well were used to extract amplitudes from each data set which were subsequently grouped to create average responses for comparison to the well data (Figure 7). Top reservoir amplitudes were generated in the well W2' (same as W2) and plotted as a blue curve. The LSM (red) and conventional migration (black) AVA curves are shown on the same figure. Without the LSM, amplitudes are misbalanced and exhibit an inaccurate AVA trend. However, LSM balances the amplitudes in the angle domain and helps to recover the AVA response. Subsequent seismic reservoir characterization benefits from the stabilized near and far amplitudes and enhanced continuity in the target section.

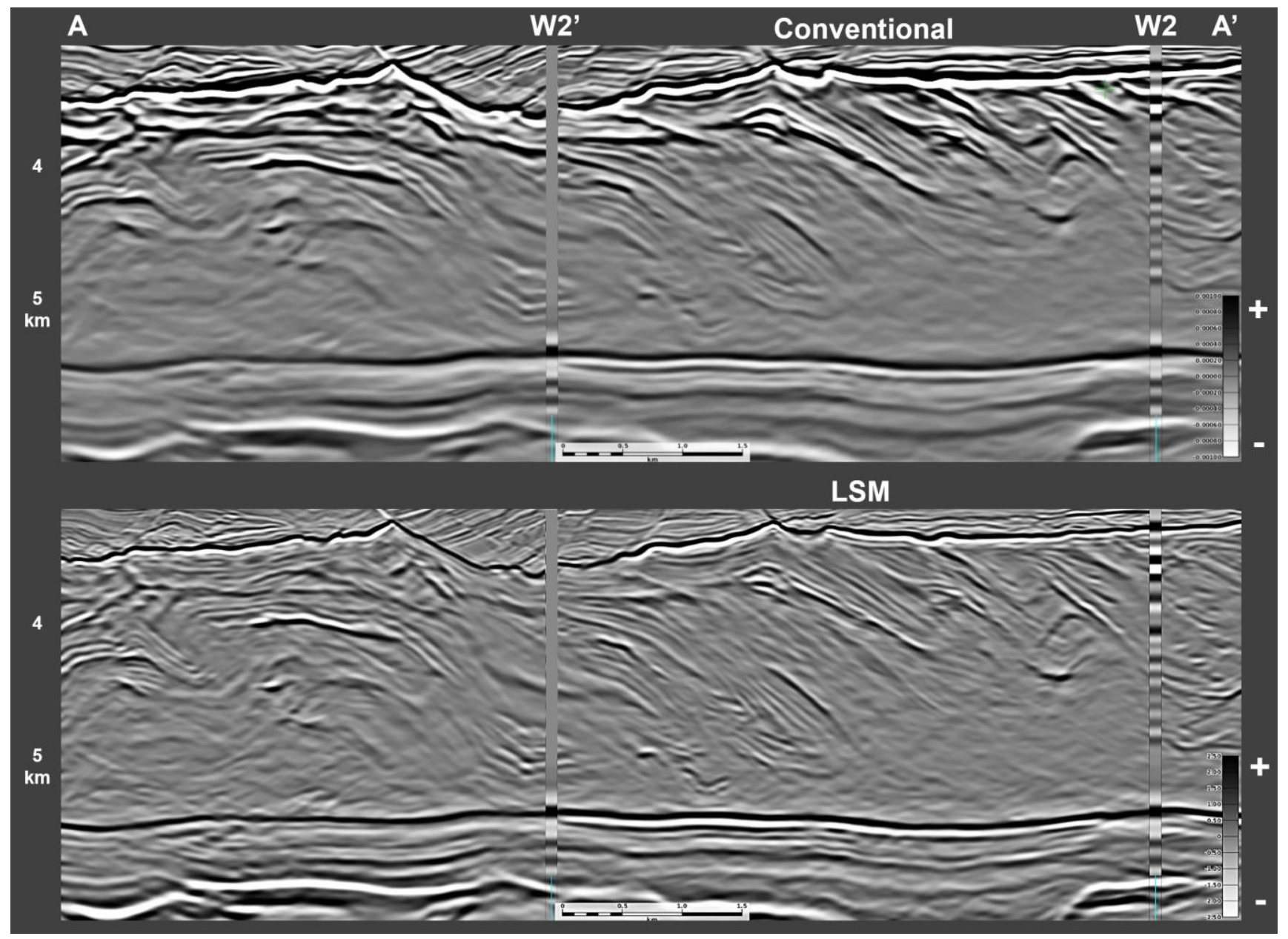

Figure 5 Full stack arbitrary line, conventional migration (top) and data-domain LSM (top). Note the improvement in the continuity and resolution of the seismic events. 


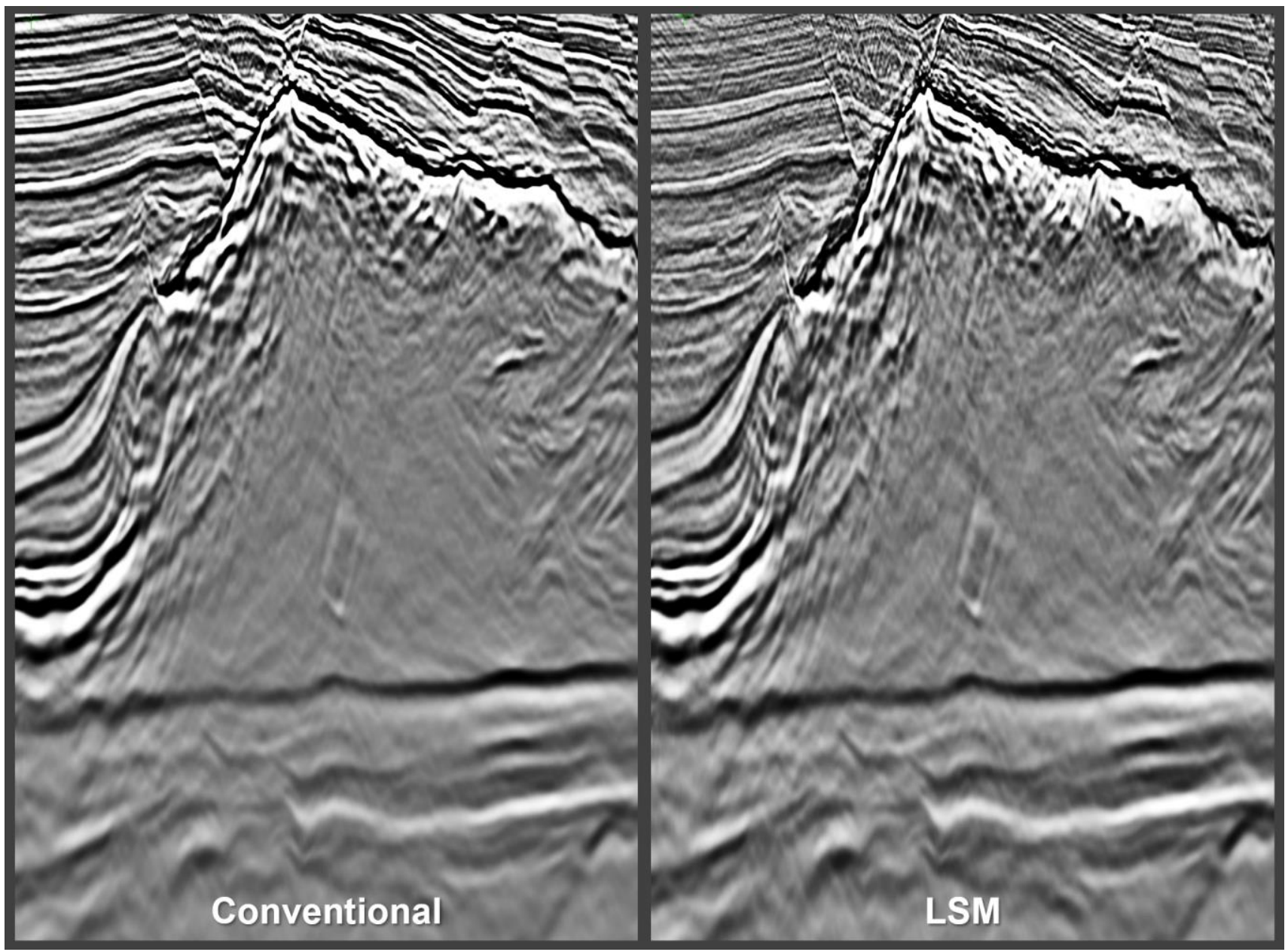

Figure 6 Conventional migration (left) and image-domain LSM (right) Far stacks.

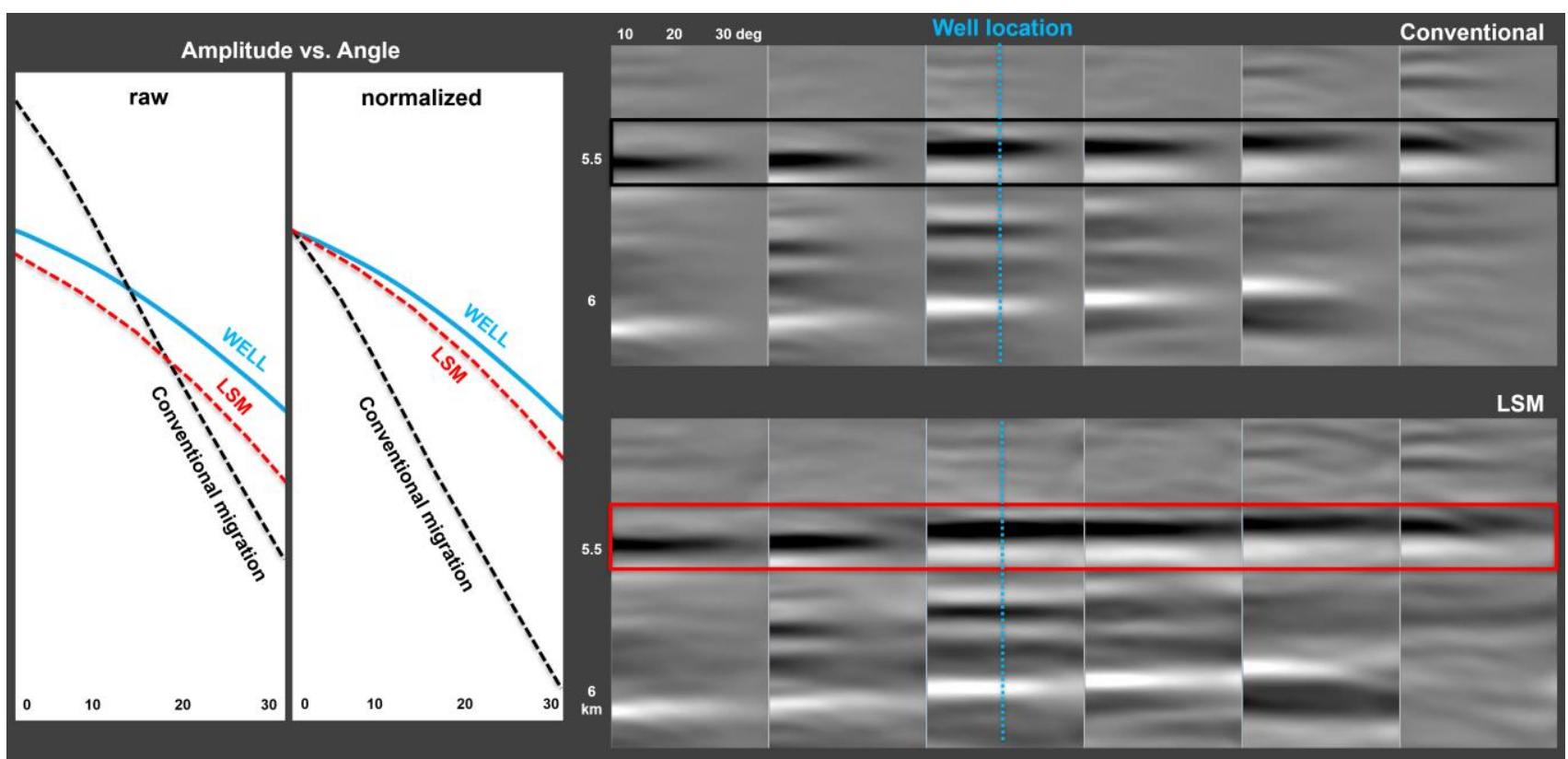

Figure 7 Angle gathers from conventional migration (top) and image-domain LSM (bottom) and their AVA plot. 


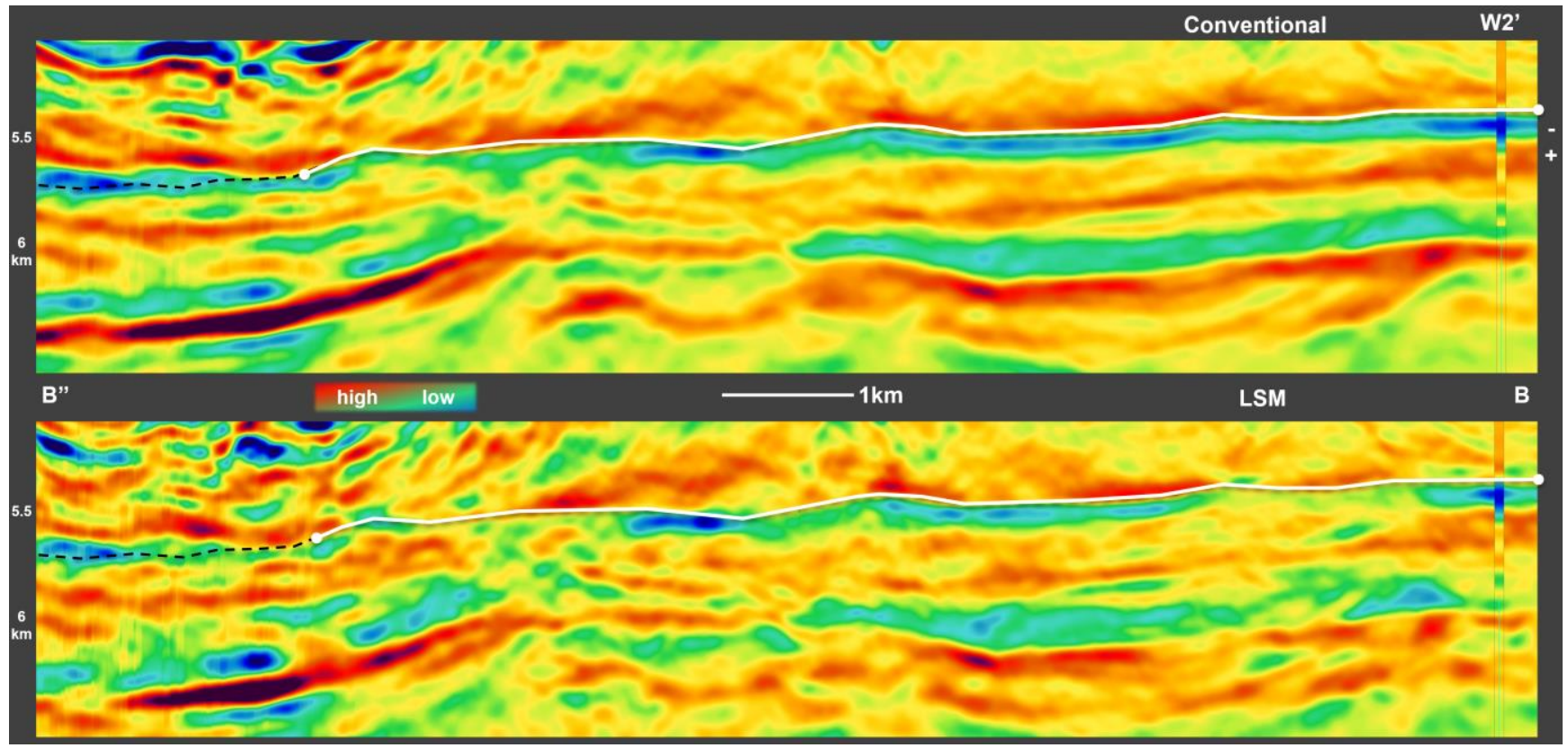

Figure 8 Relative Vp/Vs sections, conventional migration (top) and image-domain LSM (bottom).

To demonstrate the benefits of LSM, we produced relative $\mathrm{Vp} / \mathrm{Vs}$ attributes from both data sets seen in Figure 8. First note a good match at the well location (right hand side of the Figure). There are noticeable and not negligible differences in both images away from it. The white horizon denotes the extent of the Buzios field. Low $\mathrm{Vp} / \mathrm{Vs}$ values (cold colors) are expected immediately below the horizon. In the case of the conventional migration the top reservoir $\mathrm{Vp} / \mathrm{Vs}$ response seems to be steady and continuous as well as at $6 \mathrm{~km}$. However, the LSM result hints at a lateral variation in the $\mathrm{Vp} / \mathrm{Vs}$ attribute signaling the presence of zones of lateral heterogeneity. Identifying this could have a big impact on the volumetrics of this reservoir, and in general for any presalt field which has not been optimally compensated with LSM.

\section{Conclusions}

LSM has emerged as a useful tool in modern high-end data processing. The level of resolution and amplitude fidelity it provides is important for presalt seismic reservoir characterization. We have demonstrated that the presalt AVA behavior before and after LSM are different at the same $X Y$ location. Using a presalt well from Buzios field, we have been able to cross-validate the AVA curves of both migrations. The LSM result was much closer to the one observed at the well whereas, the conventional migration could not stabilize the amplitudes with angle. This suggests that the qualitative and quantitative interpretation will be more reliable with LSM data. Rock properties extracted from more stable prestack data will have less bias and will be crucial for lead and prospect de-risking, and efficient well planning.

\section{Acknowledgements}

The authors would like to thank PGS for allowing to publish this work and colleagues from R\&D and Sales and Services departments for their help and contribution.

\section{References}

S Arasanipalai et al, [2019], Innovative Imaging Technology to Unlock Presalt Reservoir Characterization. First EAGE Workshop on PreSalt Reservoirs,

Lu, S., Li, X., Valenciano, A., Chemingui, N. and Cheng, C. [2017] Least-Squares Wave-Equation Migration for Broadband Imaging. $79^{\text {th }}$ EAGE Conference and Exhibition.

Nemeth, T., Wu, C., Schuster, G.T. [1999] migration of incomplete reflection data. Geophysics, 64(1), 208221

Ramos-Martinez, J., Crawley, S., Zou, Z., Valenciano, A.A., Qui, L. and Chemingui, N. [2016] A Robust Gradient for Long Wavelength FWI Updates. $78^{\text {th }}$ EAGE Conference and Exhibition, Expanded Abstracts, SRS2

Valenciano, A.A. [2008] Imaging by wave-equation inversion. PhD thesis, Stanford University. 